\title{
Aspect ratio independent etching of dielectrics
}

\author{
Gyeong S. Hwang and Konstantinos P. Giapis \\ Division of Chemistry and Chemical Engineering, California Institute of Technology, Pasadena, \\ California 91125
}

(Received 22 April 1997; accepted for publication 27 May 1997)

\begin{abstract}
Monte Carlo simulations of pattern-dependent charging during oxide etching predict that the etch rate scaling with aspect ratio breaks down when surface discharge currents are significant. Under conditions of ion-limited etching and no inhibitor deposition, the etch depth depends on the maximum incident ion energy, reaction threshold, and surface discharge threshold, and is the same irrespective of the trench width $(\leqslant 0.5 \mu \mathrm{m})$. (C) 1997 American Institute of Physics.
\end{abstract}

[S0003-6951(97)03930-2]

When plasma etching trenches (or holes) in semiconductor or dielectric materials with a width (or diameter) below 1 $\mu \mathrm{m}$, etch rates have been observed to depend on aspect ratio (depth/width) rather than the absolute feature size. ${ }^{1}$ Several mechanisms have been invoked to explain the "rule" of aspect-ratio-dependent etching (ARDE), but no general theory has emerged that captures the variety of seemingly conflicting experimental observations reported in the literature. ${ }^{1,2}$ For example, while an ion-neutral synergy model with pure neutral flux shadowing appears to be consistent with a wealth of ARDE measurements in semiconductors, ${ }^{2}$ it does not hold for the etching of insulators. Indeed, Doemling et al. ${ }^{3}$ have reported inverse ARDE of trenches and holes in $\mathrm{SiO}_{2}$ in a high-density $\mathrm{CHF}_{3}$ plasma at 20 mTorr. Remarkably, they also reported aspect ratio independent etching (ARIE) when the pressure was lowered to $6.7 \mathrm{mTorr}$; for fixed etching time, the etch depth was the same for a variety of trench widths and hole diameters (as low as $0.25 \mu \mathrm{m}$, corresponding to an aspect ratio of 8.5:1). These authors convincingly argued that the strong influence of feature geometry on neutral flux of an etch inhibitor, produced in the $\mathrm{CHF}_{3}$ plasma, is responsible for the inverse ARDE at the higher pressure. The low pressure results were explained by hypothesizing that the neutral density at the bottom of the trench or hole, while "too low to cause inverse ARDE, it was still sufficient to suppress regular ARDE." This hypothesis must be valid for all trenches and holes etched at the low pressure, which spanned the regime of aspect ratios between 2.1:1 and 8.5:1. Bailey and Gottscho ${ }^{4}$ examined in detail the possibility of ARIE by exploiting the ability of etch inhibitors to slow down the etch rate in smaller aspect ratio trenches and concluded that this method "may be useful in minimizing ARDE but only over a limited range of aspect ratios and only with peculiar inhibitor fluxes." Even in the best of cases, with an ad hoc exponential dependence of inhibitor flux, they calculated that ARIE should break down at an aspect ratio of $\approx 5: 1$. Even if the "magic" value of the required inhibitor flux was accidentally chosen by Doemling et al., ${ }^{3}$ their smallest trench $(0.25$ $\mu \mathrm{m})$ should have been etched less deep than the larger trenches. Given the substantial reduction in inhibitor flux to the trench bottom at the low pressure, the latter argument is highly suggestive of a different mechanism responsible for ARIE up to the (impressively) high value of 8.5:1.

Differential surface charging of insulating surfaces has been suggested ${ }^{5-7}$ as a possible mechanism for ARDE during oxide etching, because local electric fields may significantly perturb ion transport in a trench, thus reducing the ion energy and flux arriving at the bottom surface. Two theoretical studies $^{6,7}$ addressed the localized charging in rectangular trenches with profound differences in the calculated potential distribution along the trench surfaces. Arnold and Sawin ${ }^{6}$ found a precipitous drop in the potential at the trench middle and speculated that the trench bottom will evolve to be concavelike. In contrast, Shibkov et al. ${ }^{7}$ calculated a potential distribution that peaks in the trench middle, suggestive of a convex trench bottom. Joubert et al. ${ }^{8}$ showed that both shapes of the bottom contours are observed, albeit as a result of the specific etch-inhibiting chemistry employed rather than charging effects. The influence of sheath dynamics on the ion and electron energy and angular distributions arriving at the wafer was not considered in these simulations. Further, surface discharge currents were neglected, despite the conjecture that these could decrease surface potentials and, thus, reduce ARDE as well. Interestingly, Shibkov et al. calculated electric fields that were very close to the breakdown threshold for bulk oxide $(1 \mathrm{MV} / \mathrm{cm})$ and argued that some surface discharge mechanisms must exist to reduce the bottom potential "because otherwise local charging would make it impossible to etch reasonably deep trenches in insulators.",

In this letter, we report results from Monte Carlo simulations of charging and ion dynamics in high aspect ratio trenches by considering the sheath oscillation effect, surface discharge currents, and validated methodology and procedures for calculating charging potentials ${ }^{9}$ with proven predictive capabilities. ${ }^{10}$ The simulation begins by calculating realistic ion and electron energy and angular distributions at the wafer from sheath theory, based on a sinusoidally varying sheath electric field. ${ }^{11}$ Then, charged particles, with translational energy and angle of approach determined by randomly sampling the corresponding distributions, are generated at a small distance above the wafer (where surface potentials decay to zero) and followed as they impinge at various surface cells, ${ }^{12}$ where they transfer their charge. Since the surface is insulating, charge deposition creates local electric fields which alter ion trajectories. The Laplace equation is solved iteratively in the simulation domain to account for the evolution of the electric fields as more charge accumulates. When the surface electric field exceeds a discharge threshold, $\widetilde{E}_{s}$, currents are allowed to flow along the surface, thereby reducing surface charging. Steady state is reached when the potential distribution at the bottom and sidewall surfaces no longer changes. At that point, a dynamic equilib- 


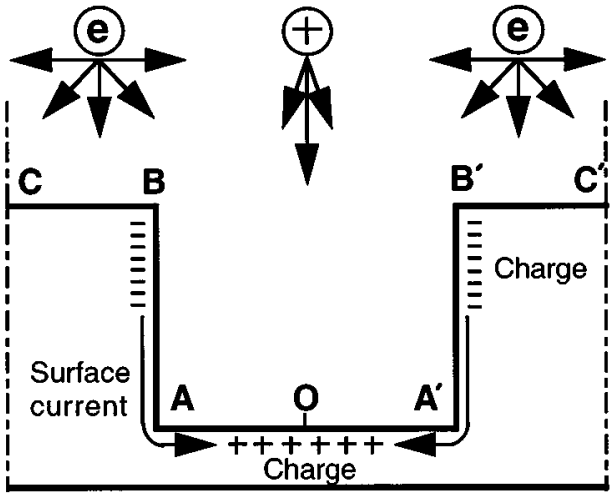

FIG. 1. Schematic of the simulation domain.

rium is established where the ion current arriving to each surface cell is balanced by electrons arriving from the plasma and neighboring cells.

The plasma conditions play a critical role in sidewall charging. Here, we employ a low-pressure $(<5$ mTorr $)$ plasma with a density of $1 \times 10^{11} \mathrm{~cm}^{-3}$; to fix the ion mass a fully ionized chlorine plasma is assumed. ${ }^{13}$ The sheath voltage is $V_{\mathrm{sh}}=0.5 V_{\mathrm{rf}}(1+\sin \omega t)+V_{\mathrm{dc}}$, where $V_{\mathrm{rf}}=50 \mathrm{~V}$ is the rf bias, $V_{\mathrm{dc}}=67 \mathrm{~V}$ is the dc sheath voltage, and $\omega=1 \mathrm{MHz}$ is the rf bias frequency. The electron and ion temperatures are taken to be 4.0 and $0.5 \mathrm{eV}$, respectively. These conditions result in bimodal ion energy distribution at the platen, similar to that expected for oxide etching. ${ }^{14}$ The geometry considered (Fig. 1) consists of equally spaced trenches (aspect ratio of $3: 1$ ), formed in a uniform dielectric. We assume that the insulator can sustain surface potential gradients of $\widetilde{E_{s}}=1$ $\mathrm{MV} / \mathrm{cm}$, a value close to the breakdown of good quality bulk oxide $\left(A\right.$ mode $\left.^{15}\right)$.

The calculated potential distributions along the trench surfaces (Fig. 2) are different for various trench widths, despite the constant aspect ratio-an indication that ARDE will not hold. The distribution for the $0.5 \mu \mathrm{m}$ trench (identical to that for a $1.0 \mu \mathrm{m}$ trench) shows some undulations at the bottom surface, with dips near the sidewalls [Fig. 2(a)]. The sidewall potential does not vary smoothly with distance because larger absolute potentials can be sustained over longer distances $(A B=1.5 \mu \mathrm{m})$ along the surface without exceeding $\widetilde{E}_{s}$. When the trench width and depth are decreased to 0.3 and $0.9 \mu \mathrm{m}$, respectively, a smoother distribution is observed along the sidewalls [Fig. 2(b)]. The distribution at the trench bottom does not change discernibly, except for a small decrease in the average potential. The trench width must decrease to $0.15 \mu \mathrm{m}$ [Fig. 2(c)] before significant changes occur. Indeed, since the trench depth is now only $0.45 \mu \mathrm{m}$, surface currents flow more readily, thus decreasing the bottom potential. Upon further decrease in the trench width and depth to 0.09 and $0.27 \mu \mathrm{m}$, respectively, the reduction in the potential becomes more dramatic. Note the similarities between the potential distribution and the topography along the trench bottom, seen experimentally: ${ }^{3}$ they are both triangular.

The decrease in the potential at the trench bottom results in large gains in the energy of ions arriving at that surface, as illustrated in Fig. 3. The initial bimodal ion energy distribution undergoes a significant change, which clearly depends

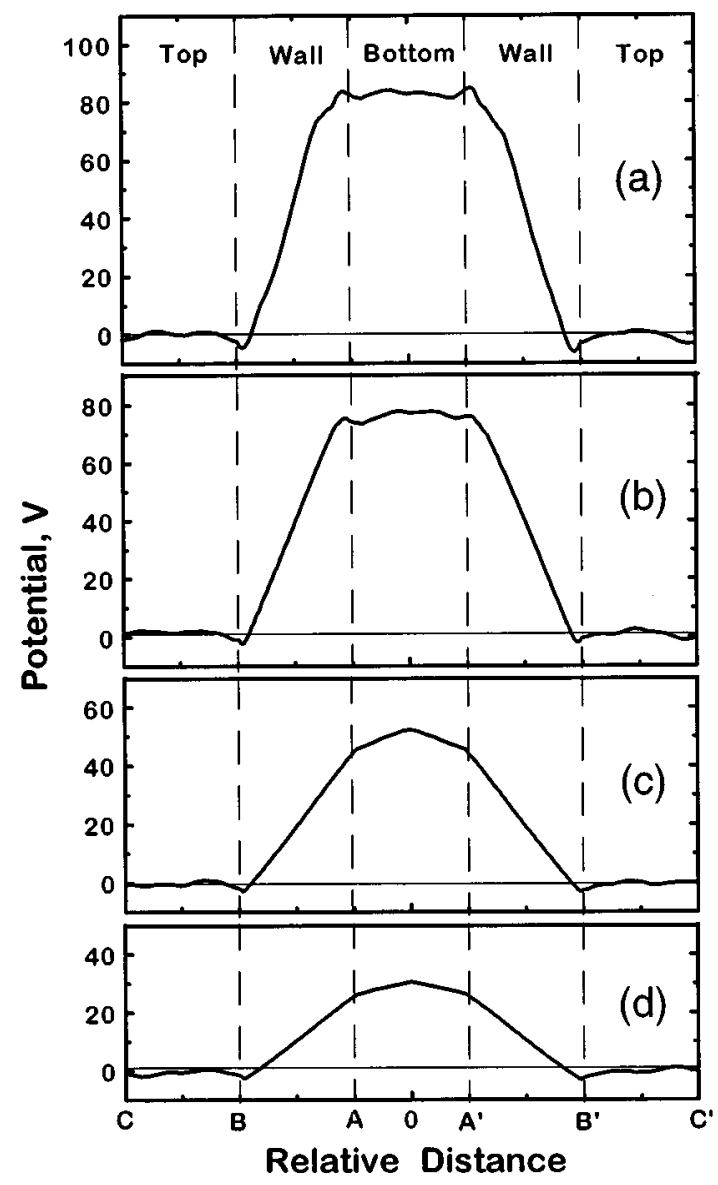

FIG. 2. Charging potential distributions along the trench surfaces as a function of the trench width $(W)$ at constant aspect ratio (3:1) and with a surface discharge threshold of $1 \mathrm{MV} / \mathrm{cm}$. Four cases are shown with $W$ : (a) 0.5 , (b) 0.3 , (c) 0.15 , and (d) $0.09 \mu \mathrm{m}$. The length scales have been normalized by the corresponding width or length of a particular segment (bottom, wall, top), to facilitate comparisons. For notation, see Fig. 1.

on the absolute trench depth since the aspect ratio is fixed. The distributions for the 1.0 and $0.5 \mu \mathrm{m}$ trenches are superimposable - the ARDE-rule holds. A large number of ions are lost to the sidewalls and the average energy decreases considerably. Upon decreasing the trench width to $0.3 \mu \mathrm{m}$, the ion energy distribution begins to shift towards larger energies - the ARDE rule begins to break down. Fi-

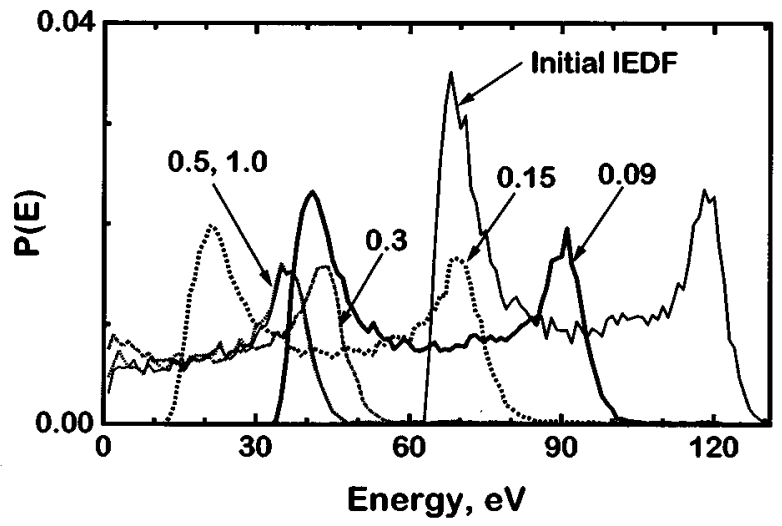

FIG. 3. The energy distribution of ions arriving at the trench bottom as a function of the trench width at constant aspect ratio (3:1) and with a surface discharge threshold of $1 \mathrm{MV} / \mathrm{cm}$. 


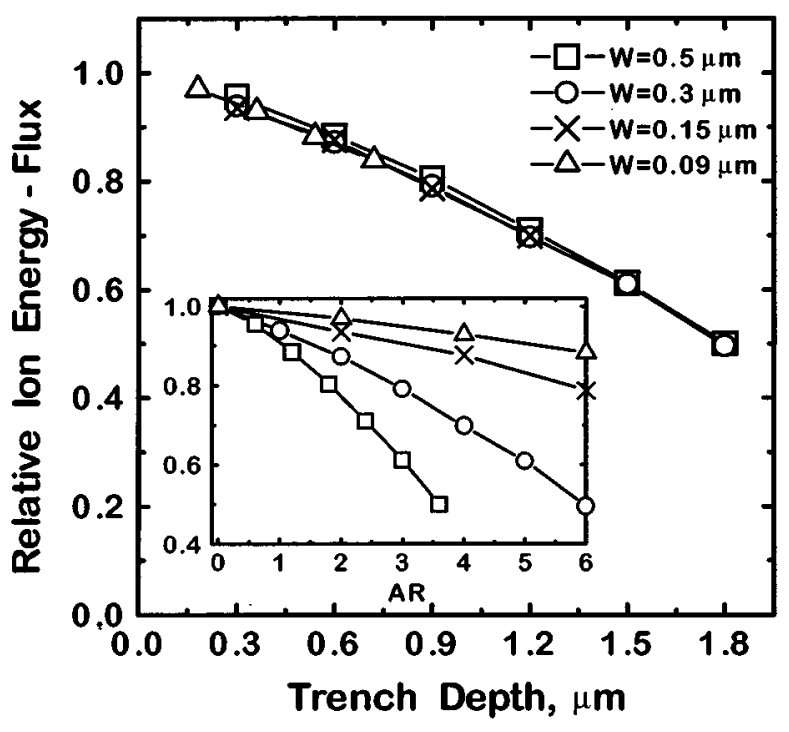

FIG. 4. The normalized average ion energy flux at the trench bottom as a function of trench depth [or aspect ratio (AR) for the inset] for various trench widths (W) as indicated. Note that sheath bias and surface discharge threshold (0.5 MV/cm) (see Ref. 17) are different from those used to obtain the distributions of Fig. 3.

nally, the distribution for the $0.15 \mu \mathrm{m}$ trench, and more so that for the $0.09 \mu \mathrm{m}$ trench, extend clearly to significantly larger energies than all previous distributions. These results suggest that the etch rate ${ }^{16}$ at the bottom of the $0.09 \mu \mathrm{m}$ trench will be much larger than that at the bottom of the 0.5 $\mu \mathrm{m}$ trench, despite the constancy of the aspect ratio. Thus, surface currents should help achieve true ARIE!

To further demonstrate that ARIE of dielectrics can be a fact, we compare in Fig. 4 the calculated average ion energyflux at the bottom of trenches of various widths as a function of the trench depth. To make this calculation relevant to oxide etching, some simulation parameters are changed as follows: (a) $\widetilde{E_{s}}=0.5 \mathrm{MV} / \mathrm{cm},{ }^{17}$ (b) ion temperature $=0.05 \mathrm{eV}$ (to eliminate ion shadowing contributions ${ }^{1}$ ), and (c) sheath potential of $V_{\mathrm{sh}}=15(1+\sin \omega t)+97 \mathrm{~V}$, where $\omega=13.56 \mathrm{MHz}$, resulting in a (relatively) monoenergetic ion energy distribution. The ion energy-flux dependence is virtually identical for all trenches of width $\leqslant 0.5 \mu \mathrm{m}$. This result strongly suggests that ARIE will be observed, provided that etching is ion limited and there is no inhibitor deposition. As the trench depth increases, larger potential differences can be tolerated; thus, surface currents cannot be as effective in reducing the bottom potential, causing a decrease in the ion energy-flux (and the etch rate ${ }^{16}$ ). Note that etching will stop once the maximum ion energy at the trench bottom decreases to the threshold value for reaction, $E_{\text {th }}\left(35 \mathrm{eV}\right.$ for the $\mathrm{CHF}_{3}$ plasma $\left.^{8}\right)$. The depth $(D)$ at which etching ceases is roughly given by $D=\left(E_{m}-E_{\mathrm{th}}\right) / e \widetilde{E}_{s}$, where $E_{m}$ is the maximum ion energy at the sheath exit, and $e$ is the electron charge. A smaller threshold will permit ARIE to greater depths, provided that the supply of neutrals to the trench bottom does not limit etching. In the extreme case of $\widetilde{E_{s}}=0$ (conductive sidewalls), the transition from ARIE to ARDE should occur at an aspect ratio where the etching switches from being ion-limited to being neutral-limited.
One question remains: Do surface discharge currents really exist? The picture of currents flowing constantly between the trench bottom and the upper sidewalls appears incredible. Strong evidence to that effect lies simply in the ability to etch high aspect ratio holes in oxide. Under low inhibitor flux deposition $\left(\mathrm{CHF}_{3}\right.$ plasma), Joubert et al. ${ }^{8}$ reported that etching ceased at an aspect ratio of 3:1 for a bias of $100 \mathrm{~V}$. Increasing bias to $150 \mathrm{~V}$ allowed etching to proceed again. Since charging steady state is rapidly reached, ${ }^{9}$ only by increasing the electron flux to the bottom surface can one achieve an increase in the ion flux and the etch rate. If surface currents did not exist, the higher ion energy would cause the buildup of larger bottom potentials so that the ion and electron fluxes balance. Since the latter is limited by shadowing (aspect ratio dependent), etching should not commence. The only other way to bring sidewall electrons to the bottom surface is by surface conduction. ${ }^{18}$

In conclusion, true ARIE of dielectric materials is possible, provided that etching is ion limited and surface discharge currents can reduce charging potentials at the bottom of trenches or holes. The depth to which ARIE occurs depends on ion energy, reaction threshold, and the surface discharge threshold; for reasonable values of these parameters $(150 \mathrm{eV}, 35 \mathrm{eV}, 0.5 \mathrm{MV} / \mathrm{cm})$ in a $\mathrm{CHF}_{3}$ plasma, ARIE to a depth of $2.3 \mu \mathrm{m}$ is predicted. The reduction of critical dimensions to $0.18 \mu \mathrm{m}$ and below should allow for a significant increase in the aspect ratio ( $>10: 1)$ before etching stops.

This material was based on work partially supported by an NSF Career Award to KPG (CTS-9623450).

${ }^{1}$ R. A. Gottscho, C. W. Jurgensen, and D. J. Vitkavage, J. Vac. Sci. Technol. B 10, 2133 (1992), and references therein.

${ }^{2}$ A. D. Bailey III, M. C. M. van de Sanden, J. A. Gregus, and R. A. Gottscho, Vac. Sci. Technol. B 13, 92 (1995).

${ }^{3}$ M. F. Doemling, N. R. Rueger, and G. S. Oehrlein, Appl. Phys. Lett. 68, 10 (1996).

${ }^{4}$ A. D. Bailey III and R. A. Gottscho, Jpn. J. Appl. Phys. 34, 2083 (1995).

${ }^{5}$ S. G. Ingram, J. Appl. Phys. 68, 500 (1990).

${ }^{6}$ J. C. Arnold and H. H. Sawin, J. Appl. Phys. 70, 5314 (1991).

${ }^{7}$ A. Shibkov, M. K. Abatchev, H. K. Lang, and M. Y. Lee, Electron. Lett. ELLEAK 32, 890 (1996).

${ }^{8}$ O. Joubert, G. S. Oehrlein, and M. Surendra, J. Vac. Sci. Technol. A 12, 665 (1994).

${ }^{9}$ G. S. Hwang and K. P. Giapis, J. Vac. Sci. Technol. B 15, 70 (1997).

${ }^{10}$ G. S. Hwang and K. P. Giapis, Appl. Phys. Lett. 70, 2377 (1997).

${ }^{11}$ M. S. Barnes, J. C. Foster, and J. H. Keller, IEEE Trans. Plasma Sci. 19, 240 (1991).

${ }^{12}$ The simulation domain is discretized by square cells; $25 \times 25$ cells $/ \mathrm{W}^{2}$ are used, where $\mathrm{W}=$ trench width.

${ }^{13}$ Since etching is not simulated, the chemistry is irrelevant in this study.

${ }^{14}$ K. Kurihara and M. Sekine, Plasma Sources Sci. Technol. 5, 121 (1996).

${ }^{15} \mathrm{~S}$. Wolf, Silicon Processing for the VLSI Era (Lattice, Sunset Beach, CA, 1995), Vol. 3.

${ }^{16}$ For ion-limited oxide etching, the etch rate is proportional to the ion energy flux, see J. Ding and N. Hershkowitz, Appl. Phys. Lett. 68, 1619 (1996).

${ }^{17} \mathrm{~A}$ value lower than $1 \mathrm{MV} / \mathrm{cm}$ is justified by the fact that doped CVD oxide-typically used in trench and via isolation-is not as good as a dielectric as thermal oxide (Ref. 15). Furthermore, it was reported in Ref. 8 that etching of $\mathrm{SiO}_{2}$ contact holes in a $\mathrm{CHF}_{3}$ plasma $\left(E_{t h}=35 \mathrm{eV}\right)$ stops at a depth of $1.5 \mu \mathrm{m}$ with $100 \mathrm{~V}$ bias; then $\widetilde{E}_{s}=(100-35) / 1.5=43 \mathrm{~V} / \mu \mathrm{m}$ or $0.43 \mathrm{MV} / \mathrm{cm}$.

${ }^{18}$ Bulk conduction or direct discharge between the upper sidewalls and the trench bottom seem implausible. Note, however, that our conclusions are not dependent on the exact electron transport mechanism, provided that there is a voltage threshold that controls the current flow. 